\title{
NUMERICAL SIMULATION OF CAVITATING TWO-PHASE GAS-LIQUID THREE-DIMENSIONAL FLOW IN A DUCT OF VARYING CROSS-SECTION BASED ON HOMOGENOUS MIXTURE MODEL
}

\author{
NGUYen The DuC \\ Institute of Mechanics, \\ Vietnamese Academy of Science and Technology
}

\begin{abstract}
The paper presents a numerical method to simulate two-phase turbulent cavitating flows in ducts of varying cross-section usually faced in engineering. The method is based on solution of two-phase Reynolds-averaged Navier-Stokes equations of two-phase mixture. The numerical method uses artificial compressibility algorithm extended to unsteady flows with dual-time technique. The discreted method employs an implicit, characteristic-based upwind differencing scheme in the curvilinear grid systems. Numerical simulation of an unsteady three-dimensional two-phase cavitating flow in a duct of varying cross-section with available experiment was performed. The unsteady important characteristics of the unsteady flow can be observed in results of numerical simulation. Comparison of predicted results with experimental data for time-averaged velocity and phase fraction are provided.
\end{abstract}

\section{INTRODUCTION}

It is highly desirable for high-speed hydraulic machinery and equipment to provide reliable operation over a wide range of operating conditions. In the estimation of performance, the existence of cavitating flow, which is considered to be high-speed gas-liquid two-phase flow, is a very important factor. Studies dealing with cavitation modeling through the computation of the Navier-Stokes equations have emerged in the recent years. These studies may broadly be classified into two categories: interface tracking models and homogeneous equilibrium flow models. In the first category, the liquid-vapor interface is tracked and grid is often regenerated iteratively to conform to the cavity region. Examples of this tracking method can be found in [1] and [2]. However, applications of the approach are limited to simple problems where cavity region can be described as a well-defined closed volume of pure gas. In addition, they are usually limited to 2-D flows because of difficulties involved in tracking 3-D interfaces. In the second category, the cavitating flow is modeled as a homogeneous two-phase mixture of liquid and vapor (see [3] and [4]). Models in this category are usually called homogenous mixture models. In comparison with interface tracking models, this approach can be easier applied to complex problems where cavity region can not be described as a well-defined closed volume of pure gas.

This paper presents the development of a three-dimensional code performing simulations of unsteady cavitating flows in ducts of varying cross-section usually faced in engineering. Its mathematical basis incorporates a homogenous mixture model and artificial compressibility algorithm. The numerical method employs an implicit, characteristicbased upwind differencing scheme in the curvilinear grid systems. This numerical method 
have been presented and applied successfully to a steady cavitating flow around a projectile [5]. In this study, an extension of artificial compressibility algorithm with dual-time technique is incorporated with the numerical method to simulate unsteady behavior of flow. Numerical simulation of unsteady three-dimensional two-phase cavitating flow in a duct of varying cross-section with available experiment is presented to show the efficiency of the code.

\section{THE COMPUTATIONAL MODEL}

\subsection{Governing equations}

In the volume fraction form, the governing equations consists of the continuity equations for vapor and liquid phases and momentum equations:

$$
\begin{gathered}
\frac{\partial \rho_{v} \alpha_{v}}{\partial t}+\frac{\partial\left(\rho_{v} \alpha_{v} u_{j}\right)}{\partial x_{j}}=-\left(\dot{m}^{+}+\dot{m}^{-}\right), \\
\frac{\partial \rho_{l} \alpha_{l}}{\partial t}+\frac{\partial\left(\rho_{l} \alpha_{l} u_{j}\right)}{\partial x_{j}}=\left(\dot{m}^{+}+\dot{m}^{-}\right), \\
\frac{\partial\left(\rho_{m} u_{i}\right)}{\partial t}+\frac{\partial\left(\rho_{m} u_{i} u_{j}\right)}{\partial x_{j}}=-\frac{\partial p}{\partial x_{i}}+\frac{\partial}{\partial x_{j}}\left[\left(\mu_{m}+\mu_{t}\right)\left(\frac{\partial u_{i}}{\partial x_{j}}+\frac{\partial u_{j}}{\partial x_{i}}-\frac{2}{3} \frac{\partial u_{k}}{\partial x_{k}} \delta_{i j}\right)\right],
\end{gathered}
$$

where $\rho_{l}, \rho_{v}, \alpha_{l}, \alpha_{v}, \dot{m}^{+}$and $\dot{m}^{-}$denote liquid density, vapor density, liquid volume fraction, vapor volume fraction, condensation rate and evaporation rate, respectively.

Mixture density and mixture viscosity can be expressed by:

$$
\begin{gathered}
\rho_{m}=\rho_{l} \alpha_{l}+\rho_{v} \alpha_{v}, \\
\mu_{m}=\mu_{l} \alpha_{l}+\mu_{v} \alpha_{v},
\end{gathered}
$$

where

$$
\alpha_{l}+\alpha_{v}=1 \text {. }
$$

By assuming that liquid density and vapor density are constants, Equations (2.1)-(2.2) can be written as follows:

$$
\begin{gathered}
\frac{\partial \alpha_{v}}{\partial t}+\frac{\partial\left(\alpha_{v} u_{j}\right)}{\partial x_{j}}=-\frac{\dot{m}^{+}+\dot{m}^{-}}{\rho_{v}} \\
\frac{\partial \alpha_{l}}{\partial t}+\frac{\partial\left(\alpha_{l} u_{j}\right)}{\partial x_{j}}=\frac{\dot{m}^{+}+\dot{m}^{-}}{\rho_{l}},
\end{gathered}
$$

For convenience, instead of Equation (2.6), the following equation obtained by adding Equation (2.7) to Equation (2.6) is employed,

$$
\frac{\partial u_{j}}{\partial x_{j}}=\left(\dot{m}^{+}+\dot{m}^{-}\right)\left(\frac{1}{\rho_{l}}-\frac{1}{\rho_{v}}\right) .
$$

Note that the differential form of the mixture density $\rho_{m}$ can be obtained by differentiating Equation (2.4) to receive,

$$
d \rho_{m}=\left(\rho_{l}-\rho_{v}\right) d \alpha_{l} .
$$

By using Equation (2.9), Equations (2.8), (2.3) and (2.7) can be written in the following vector form, 


$$
\Gamma_{e} \frac{\partial Q}{\partial t}+\frac{\partial\left(E-E_{v}\right)}{\partial x}+\frac{\partial\left(F-F_{v}\right)}{\partial y}+\frac{\partial\left(G-G_{v}\right)}{\partial z}=S
$$

where

$$
\begin{aligned}
& Q=\left[\begin{array}{l}
p \\
u \\
v \\
w \\
\alpha_{l}
\end{array}\right] ; \Gamma_{e}=\left[\begin{array}{lllll}
0 & 0 & 0 & 0 & 0 \\
0 & \rho_{m} & 0 & 0 & \left(\rho_{l}-\rho_{v}\right) u \\
0 & 0 & \rho_{m} & 0 & \left(\rho_{l}-\rho_{v}\right) v \\
0 & 0 & 0 & \rho_{m} & \left(\rho_{l}-\rho_{v}\right) w \\
0 & 0 & 0 & 0 & 1
\end{array}\right] ; S=\left[\begin{array}{l}
\left(\dot{m}^{+}+\dot{m}^{-}\right)\left(\frac{1}{\rho_{l}}-\frac{1}{\rho_{v}}\right) \\
0 \\
0 \\
0 \\
\left(\dot{m}^{+}+\dot{m}^{-}\right) \frac{1}{\rho_{l}}
\end{array}\right] ; \\
& E=\left[\begin{array}{l}
u \\
\rho_{m} u^{2}+p \\
\rho_{m} u v \\
\rho_{m} u w \\
\alpha_{l} u
\end{array}\right] ; \quad F=\left[\begin{array}{l}
v \\
\rho_{m} v u \\
\rho_{m} v^{2}+p \\
\rho_{m} v w \\
\alpha_{l} v
\end{array}\right] ; \quad G=\left[\begin{array}{l}
w \\
\rho_{m} w u \\
\rho_{m} w v \\
\rho_{m} w^{2}+p \\
\alpha_{l} w
\end{array}\right] \\
& E_{\nu}=\left[\begin{array}{l}
0 \\
\tau_{x x} \\
\tau_{x y} \\
\tau_{x z} \\
0
\end{array}\right] ; \quad F_{\nu}=\left[\begin{array}{l}
0 \\
\tau_{y x} \\
\tau_{y y} \\
\tau_{y z} \\
0
\end{array}\right] ; \quad G_{\nu}=\left[\begin{array}{l}
0 \\
\tau_{z x} \\
\tau_{z y} \\
\tau_{z z} \\
0
\end{array}\right]
\end{aligned}
$$

and

$$
\begin{array}{lll}
\tau_{x x}=\frac{2}{3}\left(\mu+\mu_{t}\right)\left(2 \frac{\partial u}{\partial x}-\frac{\partial v}{\partial y}-\frac{\partial w}{\partial z}\right) ; & \tau_{x y}=\left(\mu+\mu_{t}\right)\left(\frac{\partial u}{\partial y}+\frac{\partial v}{\partial x}\right)=\tau_{y x} ; \\
\tau_{y y}=\frac{2}{3}\left(\mu+\mu_{t}\right)\left(2 \frac{\partial v}{\partial y}-\frac{\partial u}{\partial x}-\frac{\partial w}{\partial z}\right) ; & \tau_{x z}=\left(\mu+\mu_{t}\right)\left(\frac{\partial w}{\partial x}+\frac{\partial u}{\partial z}\right)=\tau_{z x} ; \\
\tau_{z z}=\frac{2}{3}\left(\mu+\mu_{t}\right)\left(2 \frac{\partial w}{\partial z}-\frac{\partial u}{\partial x}-\frac{\partial v}{\partial y}\right) ; & \tau_{y z}=\left(\mu+\mu_{t}\right)\left(\frac{\partial v}{\partial z}+\frac{\partial w}{\partial y}\right)=\tau_{z y} .
\end{array}
$$

\subsection{Phase change closure}

The cavitation phenomenon is governed by thermodynamics and kinetics of the phase change process. Many different semi-empirical formulas have been proposed to calculate phase change rates $\dot{m}^{-}$and $\dot{m}^{+}$in Eq. (2.10). Some formulas among them have been compared in our previous study [5]. Based on the comparison results in the study [5], the formulas proposed by Anhuja et al. [4] are employed here. In these formulas, the evaporation and condensation terms are both functions of pressure and volume fraction:

$$
\dot{m}^{-}=\frac{C_{\text {dest }} \rho_{l} \operatorname{Min}\left(p-p_{v}, 0\right) \alpha_{l}}{\left(0.5 \rho_{l} U_{\infty}^{2}\right) t_{\infty}} ; \quad \dot{m}^{+}=\frac{C_{\text {prod }} \rho_{v} \operatorname{Max}\left(p-p_{v}, 0\right)\left(1-\alpha_{l}\right)}{\left(0.5 \rho_{l} U_{\infty}^{2}\right) t_{\infty}} ;
$$

where $C_{\text {dest }}=1.0$ and $C_{\text {prod }}=8.0$. 


\subsection{Turbulence closure}

In numerical simulation presented here, the turbulent viscosity $\mu_{t}$ in Eq. (2.10) is determined by standard $k-\varepsilon$ model as follows [6]:

$$
\mu_{t}=c_{\mu} \rho_{m} \frac{k^{2}}{\varepsilon} \text {. }
$$

The turbulent kinetic energy $k$ and its dissipation rate $\varepsilon$ are obtained by solving following equations,

where

$$
\begin{gathered}
\frac{\partial\left(\rho_{m} k\right)}{\partial t}+\frac{\partial\left(\rho_{m} u_{j} k\right)}{\partial x_{j}}=\frac{\partial}{\partial x_{j}}\left[\left(\mu_{m}+\frac{\mu_{t}}{\sigma_{k}}\right) \frac{\partial k}{\partial x_{j}}\right]+P_{k}-\rho_{m} \varepsilon \\
\frac{\partial\left(\rho_{m} \varepsilon\right)}{\partial t}+\frac{\partial\left(\rho_{m} u_{j} \varepsilon\right)}{\partial x_{j}}=\frac{\partial}{\partial x_{j}}\left[\left(\mu_{m}+\frac{\mu_{t}}{\sigma_{\varepsilon}}\right) \frac{\partial \varepsilon}{\partial x_{j}}\right]+c_{1} P_{k}-c_{2} \rho_{m} \varepsilon
\end{gathered}
$$

$$
P_{k}=\tau_{i j} \frac{\partial u_{i}}{\partial x_{j}} ; \quad \tau_{i j}=-\frac{2}{3} \rho_{m} k+2 \mu_{t}\left(S_{i j}-\frac{1}{3} \frac{\partial u_{k}}{\partial x_{k}} \delta_{i j}\right) ; \quad S_{i j}=\frac{1}{2}\left(\frac{\partial u_{i}}{\partial x_{j}}+\frac{\partial u_{j}}{\partial x_{i}}\right),
$$

with $c_{\mu}, c_{1}, c_{2}, \sigma_{k}, \sigma_{\varepsilon}$ are modeling constants. The constants in the standard $k-\varepsilon$ model are given as follows:

$$
c_{\mu}=0.09 ; \quad c_{1}=1.44 ; \quad c_{2}=1.92 ; \quad \sigma_{k}=1.0 ; \quad \sigma_{\varepsilon}=1.3 .
$$

\subsection{Initial and boundary conditions}

The governing equation (2.10) together with two turbulent equations (2.11) and (2.12) require initial conditions to start calculation as well as boundary conditions at every time step. In the numerical simulation presented here, the flow is assumed to start impulsively from the rest. Therefore, the uniform freestream conditions are used as initial conditions:

$$
p=p_{\infty} ; \quad u=u_{\infty} ; \quad v=v_{\infty} ; \quad w=w_{\infty} ; \quad \alpha_{l}=1.0 ; \quad k=k_{\infty} ; \quad \varepsilon=\varepsilon_{\infty} .
$$

At inlet boundary, the values of variables are also specified except that the extrapolation for pressure is applied:

$$
\frac{\partial p}{\partial n}=0 ; \quad u=u_{\infty} ; \quad v=v_{\infty} ; \quad w=w_{\infty} ; \quad \alpha_{l}=1.0 ; \quad k=k_{\infty} ; \quad \varepsilon=\varepsilon_{\infty} .
$$

At outlet boundary, the value of pressure is specified while the extrapolation for other variables is used:

$$
p=p_{\infty} ; \quad \frac{\partial u}{\partial n}=0 ; \quad \frac{\partial v}{\partial n}=0 ; \quad w=w_{\infty} ; \quad \alpha_{l}=1.0 ; \quad \frac{\partial k}{\partial n}=0 ; \quad \frac{\partial \varepsilon}{\partial n}=0 .
$$

At the solid boundaries, the no-slip condition is imposed for the velocity components and the turbulent kinetic energy $k$. The normal gradient of other variables are set to be zero:

$$
\frac{\partial p}{\partial n}=0 ; \quad u=0 ; \quad v=0 ; \quad w=0 ; \quad \alpha_{l}=1.0 ; \quad k=0 ; \quad \frac{\partial \varepsilon}{\partial n}=0 .
$$




\section{NUMERICAL METHOD}

The mass and momentum conservation equations are solved by using the dual-time extension of the artificial compressibility method [7] with the use of preconditioning technique. The transport equations of turbulent kinetic energy $k$ and its dissipation rate $\varepsilon$ (Eqs. (2.11)-(2.12)) are solved separately for each time step.

By applying the dual-time approach of the artificial compressibility method for unsteady flows and preconditioning technique [8] to Eq. (2.10), the following equation will be differentiated and solved:

$$
\Gamma \frac{\partial Q}{\partial \tau}+\Gamma_{e} \frac{\partial Q}{\partial t}+\frac{\partial\left(E-E_{v}\right)}{\partial x}+\frac{\partial\left(F-F_{v}\right)}{\partial y}+\frac{\partial\left(G-G_{v}\right)}{\partial z}=S .
$$

Here $\tau$ is the artificial time and $\Gamma$ is the preconditioning matrix formed by replacing the first column of matrix $\Gamma_{e}[9]$ :

$$
\Gamma=\left[\begin{array}{lllll}
\frac{1}{\rho_{m} \beta} & 0 & 0 & 0 & 0 \\
0 & \rho_{m} & 0 & 0 & \left(\rho_{l}-\rho_{v}\right) u \\
0 & 0 & \rho_{m} & 0 & \left(\rho_{l}-\rho_{v}\right) v \\
0 & 0 & 0 & \rho_{m} & \left(\rho_{l}-\rho_{v}\right) w \\
\frac{\alpha_{l}}{\rho_{m} \beta} & 0 & 0 & 0 & 1
\end{array}\right] .
$$

Here $\beta$ is some characteristic velocity ( $\beta$ is set to $U_{\infty}^{2}$ in numerical simulations presented here).

If Eqs. (3.1) are directly used in a Cartesian system to flow pass complex geometry, the imposition of boundary conditions will require a complicated interpolation of the data on local grid lines, since the bottom boundary of complex terrain do not coincide with coordinate lines. This leads to a local loss of accuracy in the computed solution. To avoid these difficulties, a transformation from physical domain (Cartesian coordinates $(x, y, z))$ to computational domain (generalized curvilinear coordinates $(\xi, \eta, \zeta))$ is used. In generalized curvilinear coordinates $(\xi, \eta, \zeta)$, Eqs. (3.1) can be written in the following form:

$$
\Gamma \frac{\partial \hat{Q}}{\partial \tau}+\Gamma_{e} \frac{\partial \hat{Q}}{\partial t}+\frac{\partial\left(\hat{E}-\hat{E}_{v}\right)}{\partial \xi}+\frac{\partial\left(\hat{F}-\hat{F}_{v}\right)}{\partial \eta}+\frac{\partial\left(\hat{G}-\hat{G}_{v}\right)}{\partial \zeta}=\hat{S}
$$

where $\hat{Q}=J^{-1} Q ; \hat{S}=J^{-1} S$

$$
\begin{array}{ll}
\hat{E}=J^{-1}\left(\xi_{x} E+\xi_{y} F+\xi_{z} G\right) ; & \hat{E}_{\nu}=J^{-1}\left(\xi_{x} E_{\nu}+\xi_{y} F_{\nu}+\xi_{z} G_{\nu}\right), \\
\hat{F}=J^{-1}\left(\eta_{x} E+\eta_{y} F+\eta_{z} G\right) ; & \hat{F}_{\nu}=J^{-1}\left(\eta_{x} E_{\nu}+\eta_{y} F_{\nu}+\eta_{z} G_{\nu}\right), \\
\hat{G}=J^{-1}\left(\zeta_{x} E+\zeta_{y} F+\zeta_{z} G\right) ; & \hat{G}_{\nu}=J^{-1}\left(\zeta_{x} E_{\nu}+\zeta_{y} F_{\nu}+\zeta_{z} G_{\nu}\right)
\end{array}
$$

and $J$ is Jacobian of transformation,

$$
J=\frac{\partial(\xi, \eta, \zeta)}{\partial(x, y, z)}=\left|\begin{array}{lll}
\xi_{x} & \xi_{y} & \xi_{z} \\
\eta_{x} & \eta_{y} & \eta_{z} \\
\zeta_{x} & \zeta_{y} & \zeta_{z}
\end{array}\right|
$$


Eq. (3.2) is discretized with first order finite difference for the artificial time term and the physical time term. The viscous terms are approximated by central difference expressions, while the flux splitting procedure is applied to inviscid terms [10]. The discretized equations are solved by applying an approximate factorization technique.

As mentioned, the transport equations of turbulent kinetic energy $k$ and its dissipation rate $\varepsilon$ (Eqs. (2.11)-(2.12)) are solved separately for each time step. These equations are also transformed from Cartesian coordinates to generalized curvilinear coordinates by applying the chain rule for partial derivative. The advective terms are discretized using the first-order upwind scheme. The second-order central scheme is used to discretize the diffusion-type terms. The discretized equation is solved by using an implicit approximate factorization technique. The obtained distribution of $k$ and $\varepsilon$ is utilized to update turbulent viscosity $\nu_{t}$ for the next time step. More details of solution techniques were described in [5].

\section{COMPUTED RESULT}

To test the capacity of the model, numerical simulation was performed for cavitating flow through a duct of varying cross-section with available experimental data [11]. The scheme of the experimental duct is shown in Fig. 1. The cross section of the duct is rectangular, $44 \mathrm{~mm}$ in width, and it has a variable height in order to form a convergent - divergent channel. The angle of the convergent part is $18^{\circ}$, whereas the angle at the divergent part is $8^{\circ}$. The height of the upstream section $S_{\text {ref }}$ is $45 \mathrm{~mm}$ and the height of the throat section $S_{\text {throat }}$ is $34.3 \mathrm{~mm}$.

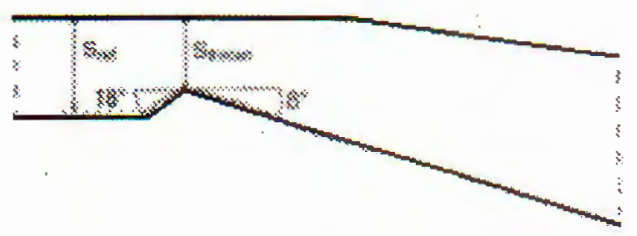

Fig.1. Scheme of the experimental duct
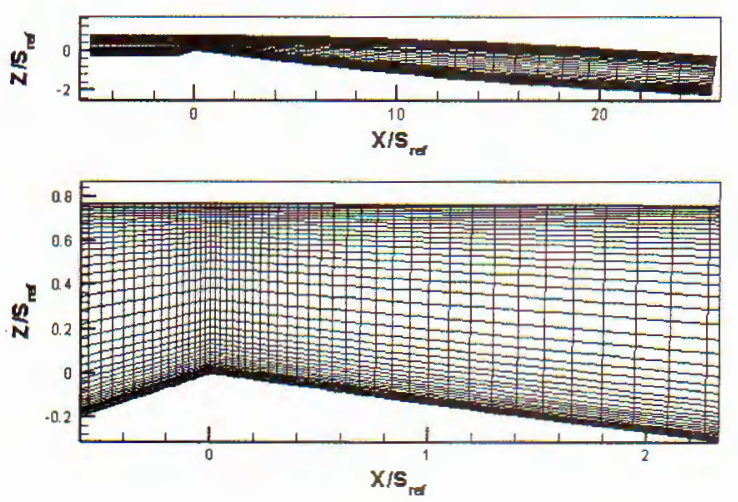

Fig. 2. Overall view (above) and close view near duct throat (below) of computation grid in a plane $\eta=$ const

The 3D grid system has 108 nodes along the streamwise direction $(\xi), 22$ nodes along the width of the duct section $(\eta)$ and 41 nodes along the height of the duct section $(\zeta)$. The 
overall and close views of calculation grid in a plane $\eta=$ const are shown in Fig. 2. The grid lines were clustered both near the solid surfaces as well as near the duct throat.

Numerical simulation was carried out for the case corresponding to an inlet velocity $U_{\infty}=7.2 \mathrm{~m} / \mathrm{s}$ and a cavitation number $\sigma=\frac{2\left(p_{\infty}-p_{v}\right)}{\rho_{\infty} U_{\infty}^{2}}=2.4$. The values at the room temperature condition: $\rho_{1}=998 \mathrm{~kg} / \mathrm{m}^{3} ; \rho_{\nu}=998 \mathrm{~kg} / \mathrm{m}^{3}$ and $\nu=1.004 \times 10^{-6} \mathrm{~m}^{2} / \mathrm{s}$ are used in the simulation. The simulation is performed with the nondimensional time step $\Delta t^{*}=\frac{\Delta t}{T_{\text {ref }}}=0.005$. Here $T_{\text {ref }}$ is the reference time associated with the reference velocity $U_{\infty}$ and the reference length $S_{\text {ref }}\left(T_{\text {ref }}=\frac{S_{\text {ref }}}{U_{\infty}}\right)$. After about 2000 time steps of simulation, a self-oscillatory flow were obtained. The cavitation zones appears as vapor clouds near the bottom surface just after the duct throat. The analysis of computed results shows a more or less regular shedding of vapor clouds that are convected downstream and then collapsed. The transient evolution of flow is almost periodic with its cycles which can be described as follows:

1. For about one haft of the cycle, a vapor cloud grows. This vapor cloud is almost attached to bottom surface.

2. The length of attached vapor cloud increases. At the same time, a second separate vapor cloud is convected downstream.

3. A disturbance appears at the interface of the attached vapor cloud when the cloud starts to be collapsed.

4. The attached vapor cloud then split in to two parts while the downstream second vapor cloud start to disappear.

The views of cavitation zone at some different time in a cycle corresponding to four above stages can be shown in Fig. 3 .

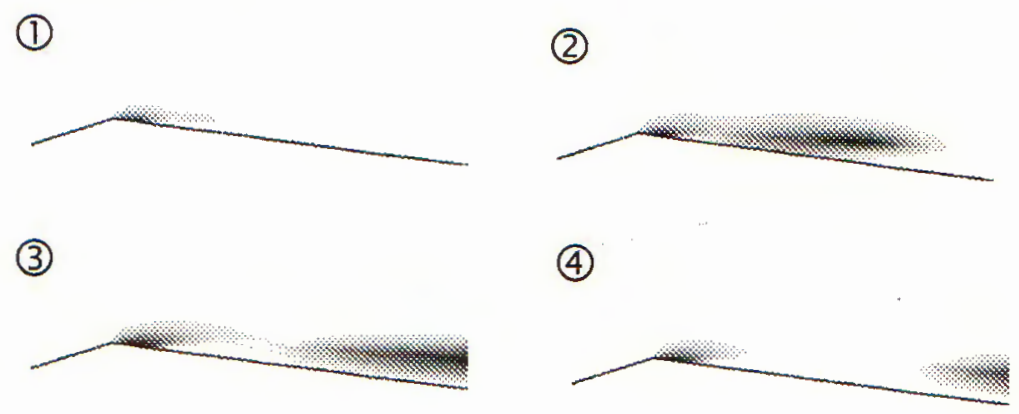

Fig. 3. Distribution of vapour volume fraction at some different times in a cycle of computed flow

This semi-periodic behavior of computed cavitating flow totally agree with experimental observation. The numerical simulation also indicates the development of a reversed jet near the bottom solid surface. When this reversed jet reaches to the interface, the attached vapor cloud splits into two parts. The existence and position of the reversed jet in flow can be observed in Fig. 4. 

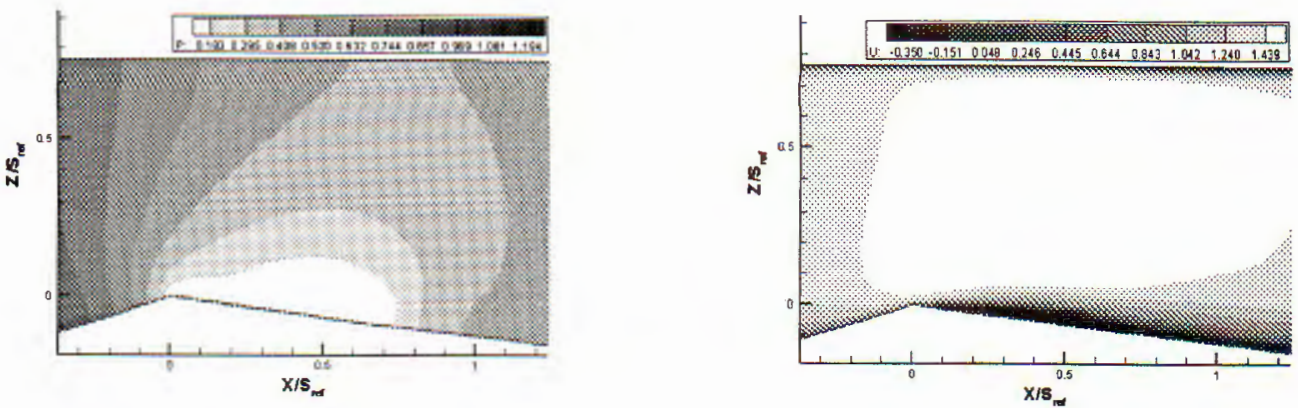

Fig. 4. Computed distributions of pressure (left) and velocity component $U$ (right) at a time when the breakoff of attached vapour cloud starts to occur

It is clear that some most important behaviors of experimental flow have been simulated by the numerical model. In order to provide further test of the capacity of the simulation method, some quantitative comparisons between measurements and computed results were also made. In the experiment, taps are provided on the bottom of the duct at the distances of $13.7 \mathrm{~mm}, 31.5 \mathrm{~mm}, 49.9 \mathrm{~mm}$ and $67.7 \mathrm{~mm}$ downstream of the throat. They are equipped with optical probes to produce measurements of velocity and vapor volume fraction. These measured values were reported in the time-averaged form. The measurement techniques and result are present in detail in [11]. The time-averaged velocity component $U$ are presented for each profile in Fig. 5. It shows the quite good agreement between numerical and experimental results in two positions (b) and (c). However, the poorer agreement can be seen in the positions (a) and (d). The position (a) corresponds to the front part of cavitation zone where the evaporation process starts to occur.

(a)

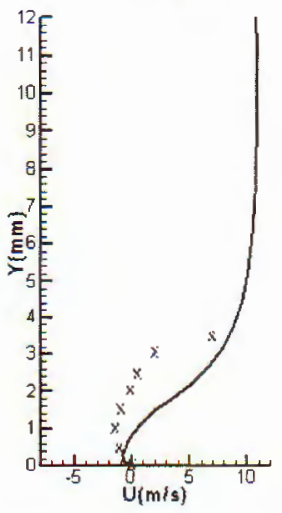

(b)

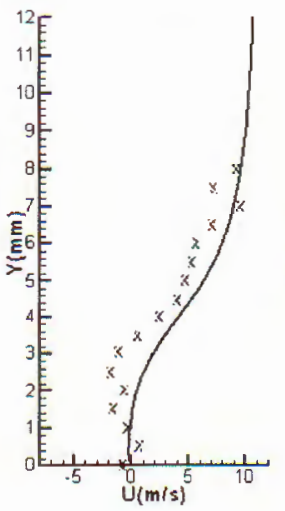

(c)

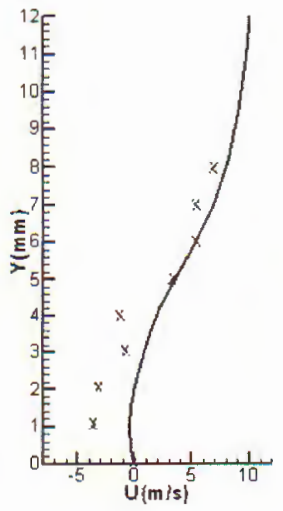

(d)

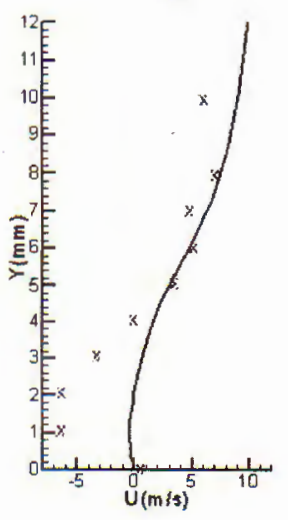

Fig. 5. Time-averaged velocity component $U$. Comparison between numerical result (lines) and measurements (points) at the distances of $13.7 \mathrm{~mm}$ (a), $31.5 \mathrm{~mm}$ (b), $49.9 \mathrm{~mm}$ (c) and $67.7 \mathrm{~mm}$ (d) downstream of the throat

The position (d) corresponds to the rear part of cavitation zone where the evaporation process together with the condensation process occurs simultaneously. The computed and measured profiles of the time-averaged vapor volume fraction $\alpha_{v}$ can be shown in Fig. 6. It can be seen that the agreement between numerical simulation and experiment in the case of the volume fraction is better than in the case of the velocity component $U$. 
The computed vapor volume fraction profiles are in quite good qualitative agreement with measurements in three positions (a), (b) and (c). The poorer agreement can be seen in the position (d).

From the quantitative comparisons presented in Figs. 5 and 6, it can be concluded that, in general, the quite good agreements with the experiment of the numerical simulations are obtained in the center parts of cavitation zone while the poorer agreements are faced at the front and rear parts. In author's opinion, this difference may be related to the fact that the local speed of sound in the front and rear parts of cavitation zone (where liquid and vapor phases are mixed strongly) is smaller than the local speed of sound in the center parts (nearly pure vapor). The larger local Mach number in the front and rear parts leads to the poorer agreement between numerical simulation and experiment because the incompressible model presented here is more suitable with flows with small Mach number.

(a)

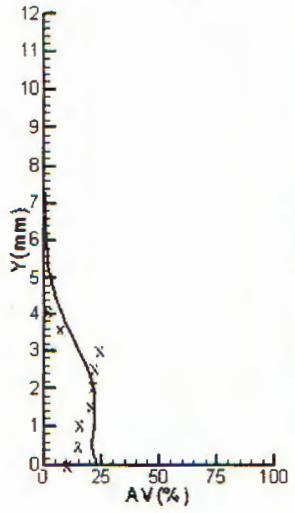

(b)

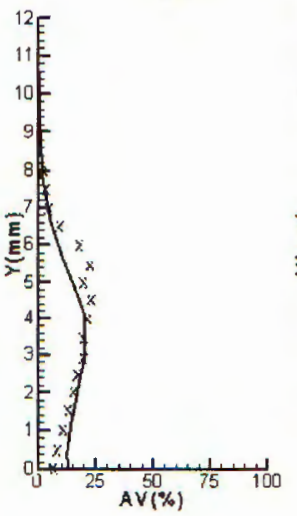

(c)

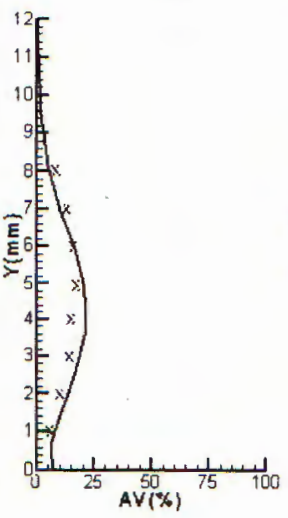

(d)

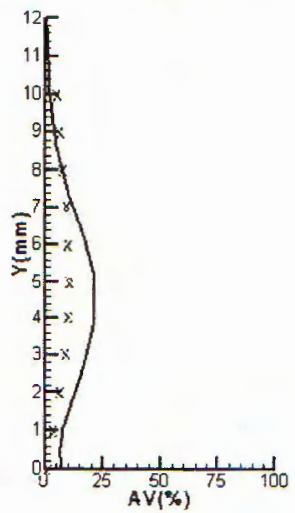

Fig. 6. Time-averaged vapour volume fraction $\alpha_{v}$. Comparison between numerical result (lines) and measurements (points) at the distances of $13.7 \mathrm{~mm} \mathrm{(a),} 31.5 \mathrm{~mm}$ (b), $49.9 \mathrm{~mm}$ (c) and

$67.7 \mathrm{~mm}$ (d) downstream of the throat

\section{CONCLUSION}

A numerical method to simulate two-phase turbulent cavitating flows in ducts of varying cross-section usually faced in engineering was presented. The method is based on solution of the incompressible two-phase Reynolds-averaged Navier-Stokes equations of two-phase mixture. A code have been developed based on the numerical solution of the governing equations.

The qualitative and quantitative comparisons between numerical and experimental results have been performed. The qualitative comparisons show that most important characteristics of experimental flow have been simulated by the numerical model. The semi-periodic behavior of computed cavitating flow totally agree with experimental observation. The numerical simulation also indicates the development of a reversed jet near the bottom solid surface. From the quantitative comparisons, it can be concluded that the quite good agreements with the experiment of the numerical simulations are obtained in the center parts of cavitation zone while the poorer agreements are faced at the front and rear parts. The poorer prediction in the front and rear parts of cavitation zone may be related to the large local Mach number in these parts where two phase are strongly 
mixed which causes the incompressible model presented here becomes less appropriate. The use of a compressible model seem to be a reasonable alternative. However, because much larger parts of cavitating flows still have small local Mach number, the application of a compressible model will require strong computing resources and will be inappropriate in the condition of our computer at the present and in the near future.

The study shows that the present numerical method can be used to predict unsteady cavitating flows in technical ducts of fluid machinery. It is clear that the method should be used together with simpler methods in order to provide more detailed information of unsteady cavitating flows faced in engineering.

Acknowledgment. This publication is completed with the financial support from National Basic Research Program in Natural Sciences

\section{REFERENCES}

1. A. Kubota, H. Kato, H. Yamaguchi, Cavity Flow Prediction Based on Euler Equations, J. Fluids Mech. 240 (1992) $59-96$.

2. M. Deshpande, J. Feng, C. L. Mercle, Numerical Model of Thermodynamics Effects of Cavitation, J. Fluids Eng. 119 (1997) 420 - 427.

3. J. Lindau, R. Kunz, D. Boger, D. Stinerbring, H. Gibeling, High Reynolds Number, Unsteady, Multiphase CFD modeling of Cavitating Flow, J. Fluids Eng. 124 (2002) 607-615.

4. V. Ahuja, A. Hosangadi, S. Arunajatesan, Simulation of Cavitating Flows using Hybrid Unstructured Meshes, J. Fluids Eng. 123 (2001) 331 - 340.

5. Nguyen The Duc, W. G. Park, A Numerical Navier-Stokes Solver for Turbulent Cavitating Flow Computation, Proceedings of the $2^{\text {nd }}$ Asia-Pacific Workshop on Marine Hydrodynamics, Busan, June 21-22, 2004, pp. 85-90.

6. D. C. Wincox, Turbulence Modeling for CFD, DCW Industries Inc, La Canada, California, USA, 1993.

7. A. J. Chorin, A Numerical Method for Solving Incompressible Viscous Flow Problem, Journal of Computational Physics 2 (1967) 12 - 26.

8. E. Turkel, Preconditioning Techniques in Computational Fluid Dynamics, Annual Review Fluid Mechanics 31 (1999) 385 - 416.

9. S. Venkateswaran et al., Preconditioning Algorithms for Computation of Multi-Phase Mixture Flows, AIA A Paper 2001-0279, 2001.

10. E. F. Toro, Riemann Solvers and Numerical Methods for Fluid Dynamics - A Practical Introduction, Springer-Verlag, Berlin, 1999.

11. B. Stutz and J. L. Reboud, Experiments on unsteady cavitation, Experiment in Fluid 22 (1997) 191 - 198. 


\section{MÔ PHỎNG SỐ DÒNG BA CHIỀU HAI PHA DO XÂM THỰC TRONG ỐNG CÓ TIẾT DIỆN KHÔNG ĐỀU TRÊN CƠ SỞ MÔ HİNH HỖN HợP DỒNG NHẤT}

Bài báo trình bày một phương pháp mô phơng số dòng xâm thực rối hai pha trong các ống có thiết diện không đều thường gặp trong kỹ thuật. Phương pháp dựa trên lời giải của hệ phương trình Navier-Stokes trung bình Reynolds cho hỗn hợp hai pha. Phương pháp giải sử dụng thuật toán nén giả được mở rộng cho dòng không dừng với kỹ thuật hai bước thời gian. Phương pháp rời rạc hóa sử dụng một hệ sai phân ẩn, ngược dòng theo đường đặc trưng trong các hệ lưới cong. Mô phỏng số được thực hiện cho một dòng xâm thực hai pha ba chiều không dừng trong một ống có thiết diện không đều và kết quà được so sánh với số liệu thí nghiệm. Các đặc trưng không dừng quan trọng của dòng có thể được quan sát trong kết quà mô phơng số. So sánh kết quả tính toán với dữ liệu thí nghiệm về vận tốc và tỷ phần pha trung bình theo thời gian cũng được thực hiện. 